\title{
Cost-Effective, Cognitive Undersea Network for Timely and Reliable Near-Field Tsunami Warning
}

\author{
X. Xerandy ${ }^{\dagger}$, Taieb Znati ${ }^{\ddagger}$, Louise K Comfort ${ }^{ \pm}$ \\ ${ }^{\dagger}$ School of Information Sciences \\ ${ }_{\text {†Computer Science Department }}$ \\ ${ }^{ \pm}$Graduate School of Public and International Affairs \\ University of Pittsburgh, Pittsburgh
}

\begin{abstract}
The focus of this paper is on developing an early detection and warning system for near-field tsunami to mitigate its impact on communities at risk. This a challenging task, given the stringent reliability and timeliness requirements, the development of such an infrastructure entails. To address this challenge, we propose a hybrid infrastructure, which combines cheap but unreliable undersea sensors with expensive but highly reliable fiber optic, to meet the stringent constraints of this warning system. The derivation of a low-cost tsunami detection and warning infrastructure is cast as an optimization problem, and a heuristic approach is used to determine the minimum cost network configuration that meets the targeted reliability and timeliness requirements. To capture the intrinsic properties of the environment and model accurately the main characteristics of the sound wave propagation undersea, the proposed optimization framework incorporates the Bellhop propagation model and accounts for significant environment factors, including noise, varying undersea sound speed and sea floor profile. We apply our approach to a region which is prone to near-field tsunami threats to derive a cost-effective under sea infrastructure for detection and warning. For this case study, the results derived from the proposed framework show that a feasible infrastructure, which operates with a carrier frequency of $12-\mathrm{KHz}$, can be deployed in calm, moderate and severe environments and meet the stringent reliability and timeliness constraints, namely 20 minutes warning time and $99 \%$ data communication reliability, required to mitigate the impact of a near-field tsunami. The proposed framework provides useful insights and guidelines toward the development of a realistic detection and warning system for nearfield tsunami.
\end{abstract}

Keywords-near field tsunami, undersea, sensor, fiber optic, detection, optimization, cost, reliable, timeliness

\section{INTRODUCTION}

Tsunami is a series of seismic sea waves, usually generated by disturbances associated with earthquakes occurring below or near the ocean floor, volcanic eruptions, submarine landslide and coastal rock falls [1]. Tsunami waves are of extremely long length and period. Based on the distance of the tsunami source to the coast, or alternatively the coast travel time, a tsunami can be classified as local, regional or distant. A local tsunami, also referred to as near-field tsunami (NFT), originates at a nearby source, typically $100 \mathrm{~km}$ (or less than 1 hour travel time). Its destructive effects are confined to the coast within this distance. A regional tsunami originates from within about 1000 $\mathrm{km}$ from the coast. It is capable of massive destruction within a geographic region where it occurs, although it may occasionally inflict a limited and localized destructive effect outside the region. A distant tsunami, also referred to as far-field tsunami, originates from a far away source, generally more than 1000 $\mathrm{km}$ away from the coast. Although less frequent than local and regional tsunami, distant tsunami causes extensive destructions near the source and has sufficient energy to cause additional destruction on shores more than $1000 \mathrm{~km}$ away from the source. A study of tsunami in the Pacific region, shows that $87 \%$ tsunamigenous sources are located closer than $100 \mathrm{~km}$ from the coast [2]. Reliable detection of a near-field tsunami becomes critical to ensure timely evacuation and prevent loss of lives and severe damage.

Several systems were developed for early tsunami detection. NOAA developed and deployed Deep-ocean Assessment and Reporting of Tsunami (DART) [3]. DART consists of a sea floor bottom pressure recorder and a moored surface buoy. An acoustic link connects the recorder to the buoy for real time communication of temperature and pressure data. The buoy sends the data to the land station through satellite communication. However, DART buoy is primarily suitable to detect far-field tsunami, as they are deployed and anchored at least 250 miles away from the shore [4]. In addition to damage caused by weather condition, floating buoys may also be subject to vandalism, particularly in marine water that is favorable to fishing [5]. This makes servicing damaged buoys challenging and costly. The ocean-bottom seismographic and tsunami observation system, based on fiber-optics submarine cable, was also developed for tsunami detection [6]. It monitors sea floor earthquake to detect tsunami over higher accuracy and finer dynamic range. However, this system comes with prohibitively high deployment cost and may not be affordable for third world countries.

This paper focuses on near-field tsunami and seeks to develop a cost effective tsunami detection system for reliable delivery of warnings to ensure timely evacuation. To this end, a hybrid, buoy-less underwater communication infrastructure is proposed. The infrastructure is composed of a fiber optic and an undersea network of sensors. Fiber optic provides high reliability and fast data transmission. Its acquisition, deploy- 
ment and maintenance costs may be prohibitive. Undersea sensors have low costs and require minimal maintenance. However, underwater sensor technology, which uses acoustic channels for communication, presents several challenges. Although acoustic channels offer longer transmission ranges than radio waves, they have high latency and limited capacity due to time-varying multi-path propagation. They are also susceptible to interference caused by environment factors, such as sea bottom shape and material, noise, sea temperature and salinity. This makes reliable and timely message delivery a difficult challenge.

To address the challenges the design of a hybrid underwater infrastructure entails, we explore the trade-offs between costeffectiveness, reliability and tsunami warning delivery timeliness. To this end, we propose an optimization framework and use this framework to derive a nearly-optimal underwater communication infrastructure that achieves the specified transmission reliability and meets the warning time delivery constraint. In this framework, we use the Bellhop model to correctly capture the characteristics and propagation behavior of an acoustic communications channel, including transmission loss and the ray's arrival times [7]. We also incorporate required sea environment data such as sea bottom profile and sound speed profile into the model, and apply Wenz curve [8] to approximate sea noise intensity.

Within this framework, the design of a cost-effective, reliable hybrid underwater network is formulated as a costoptimization problem, subject to the specified reliability and time delivery constraints needed to ensure timely evacuation, upon the detection of a tsunami. Incorporating complex models to capture the behavior of an acoustic channel, combined with the time-varying nature of the environment, makes the formalized problem NP hard. We use a heuristic approach to develop near-optimal solution to this problem. The heuristic iteratively explores the trade-off between the length of the fiber optic cable and the number of sensors needed to enable the hybrid communications infrastructure that meets the reliability and timing constraints. In order to compute the optimal distance between two adjacent sensors and compare it to the cost of the equivalent fiber optic segment, the Matlab ${ }^{\mathrm{TM}}$ optimization toolbox, together with Bellhop application suit [7], is used. The developed heuristic is used to explore a cost-effective. hybrid infrastructure suitable to detect near-field tsunamis. More specifically, the main contributions of the paper are summarized below:

- A hybrid infrastructure for cost effective, reliable and timely near-field tsunami detection which uses undersea sensor network and fiber optic.

- An optimization framework that incorporates accurate acoustic channel behavior and sea environmental factors.

- A heuristic approach to derive a practical and costeffective, near-optimum underwater communications infrastructure for near-field tsunami detection and timely warning delivery.

- The application of the developed heuristic to develop a hybrid infrastructure for the near-field tsunami prone city of Padang, West Sumatra, Indonesia [9]. The analysis of the derived infrastructure is carried out and several sce- narios, under different design parameters, are explored.

The remainder of the paper is organized as follows: Section II discusses the proposed topology and provides a description of the communication components of the hybrid network. Section III presents the optimization framework, including the formulation of the cost-effective hybrid network design problem, subject to the reliability and the timeliness constraints. Section IV describes, in detail, the proposed heuristic approach to obtain a near-optimal solution to the network design problem. Section V discusses the application of the heuristic to the design of a cost-effective undersea communications infrastructure for the city of Padang. The performance analysis of the proposed infrastructure, for different design parameters, is also presented. The final section provides the conclusion of this work and discusses future research directions.

\section{NEAR-FIELD TSUnAMI WARNING SySTEM}

To address near-field tsunami potential threat, we propose a hybrid infrastructure, composed of a bottom pressure sensor, acoustic relay sensors and a fiber optic communication link.

The bottom pressure sensor, which is the closest to the epicenter, comprises three functional modules, namely a pressure sensing module, bottom pressure recording module and an acoustic communication module. It can operate either in standard or event mode. In standard mode, the bottom pressure sensor routinely senses, records, and transmits the recorded data at regular time intervals. Upon the detection of an event, such as changes in bottom sea pressure, the bottom pressure sensor enters the event mode and increases its transmission rate until no further events are detected [3].

A relay sensor consists of an acoustic communication module, which is capable of transmitting and receiving data. Acoustic links operate in half-duplex communication mode. Linked together, they provide a multi-hop communication from the bottom pressure sensor to the optic fiber communication link. An undersea gateway node, attached to the fiber optic cable, is required to convert the received acoustic signal into an optical signal. The received information is forwarded to the control station ashore, for tsunami detection warning dissemination. Fig 1 illustrates the infrastructure.

The proposed system must deliver information reliably and in a timely manner, in order to meet the tsunami preparedness and response requirements. Furthermore, the infrastructure cost must be minimized in order to achieve deployment at scale. To this end, the system design must address two critical constraints, namely reliability and timeliness. The reliability constraint specifies that the data loss probability should not exceed a target threshold. Note that to send data over acoustic link reliably, the acoustic signal must be interpretable at the receiving node. This imposes a limit on the maximum distance between any two adjacent sensor nodes in the hybrid infrastructure. The timeliness constraint sets an upper bound on the delay of a message from the pressure sensor to the onshore station. This is necessary to deliver the tsunami warning on time for the disaster response managers to organize the evacuation plan.

In addition to acoustic loss due to propagation, sea ambient noise also contributes to acoustic signal quality at the receiving 


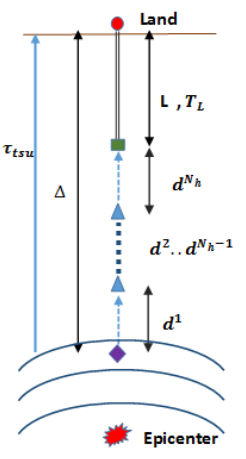

(a)

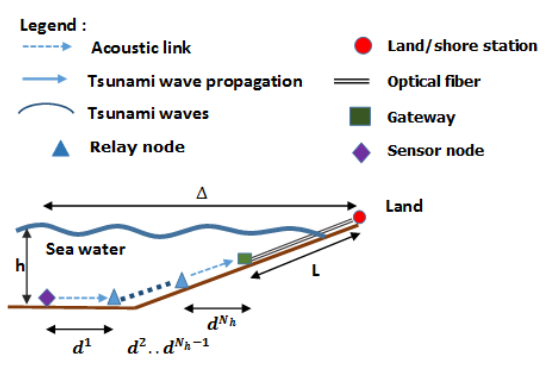

(b)
Fig. 1: The topology for notational convention

node. Consequently, the need to accurately compute the acoustic transmission loss becomes critical in order to meet the two main constraints of the the proposed optimization framework. To this end, we use Bellhop propagation model to determine acoustic transmission loss. We further incorporate the sea floor and underwater sound speed profiles to take into consideration the characteristic of the environment.

\section{OPTIMIZATION FRAMEWORK FORMULATION}

Figures 1(a) and 1(b) depict the proposed architecture viewed from the top and the side perspective, respectively. The legend of notations used in Fig. 1 is provided in table I.

The optimization framework is formulated as a minimization of infrastructure cost subject to the timeliness and reliability constraints. Let $C_{t o t}$, which depends on $N_{h}, \Delta$, and $\boldsymbol{d}$, denotes the implementation cost. The minimization problem can be stated as follows:

$$
\text { Minimize } C_{\text {tot }}(\Delta, \boldsymbol{d})=C_{F}(\Delta, \boldsymbol{d})+C_{S}\left(N_{h}\right)
$$

Subject to

$$
\begin{aligned}
T_{W a r n}(\Delta, \boldsymbol{d}) & \geq \Theta_{T} \\
R^{i}(\Delta, \boldsymbol{d}) & \geq \Theta_{R} ; \quad i=1,2, . . N_{h}, \\
R^{F}(\Delta, \boldsymbol{d}) & \geq \Theta_{R} \\
L=\Delta-\sum_{i=1}^{N_{h}} d^{i} & \geq L_{\min } ; \\
\Delta & \leq \Delta_{\max }
\end{aligned}
$$

where $\boldsymbol{d}=\left\{d^{i} \mid d^{i}>0,1 \leq i \leq N_{h}\right\}$. The timeliness and reliability requirements are represented by Eq. (1), Eq. (2), and Eq. (3), respectively.

In practice, a submarine fiber optic cable is used to connect the last undersea acoustic link to the central station onshore via an optic fiber gateway attached to the fore front of the cable. As the transmission over acoustic links approaches coastal area, the sea depth becomes shallower, thereby impeding transmission between sensor nodes. Furthermore, there may be a requirement that a minimum length of the fiber optic to be deployed offshore be buried underneath the sea floor. This requirement is usually imposed by local government regulations in order to avoid cable damage due to ship's anchor drop-off around coastal areas. To meet this requirement, an auxiliary constraint on the length of the fiber, $L$, must be added. This constraint is expressed in Eq. (4). The Eq. (5) expresses the fact that the distance of the pressure sensor from the onshore station should not exceed the epicenter. In the context of near-field tsunami, the value of $\Delta_{\max }$ obviously doesn't exceed the distance defined for near-field tsunami origin.

Because of the environment variability, in terms of sea floor profile and ambient noise characteristics, sensor nodes may not be equidistant. Therefore, the total number of the undersea nodes, $N_{h}$, which depends on the cost trade-off between optic fiber and sensor nodes, cannot be determined a priori. A similar observation can be made with respect to Eq. (4), as $\sum_{i=1}^{N_{h}} d^{i}$ cannot simplify into $N_{h} \cdot d$, which would have been the case if all sensor nodes were equidistant. The variability of $N_{h}$ further compounds the complexity of the optimization problem.

In the following, we introduce the cost model used in this optimization problem and elaborate on the timeliness constraint. We then derive the data delivery time and the link reliability.

\section{A. Total Cost Formulation}

In this framework, a linear cost function is used. Based on this function, the total cost, $C$, to deploy $Q$ units, can be expressed as $C=\phi Q+C_{0}$, where $\phi$ is the unit cost and $C_{0}$ is the initial cost. It is worth noting, however, that the framework does not depend on a specific cost function. Other functions such as power-law and logarithmic could have been used.

\section{TABLE I: Notation explanation}

\begin{tabular}{l|l} 
Notation & Remark \\
\hline$C_{t o t}$ & Total deployment cost \\
$C_{F}$ & Cost to deploy fiber \\
$L$ & Length of fiber \\
$L_{\text {min }}$ & Minimum fiber optic length \\
$\Delta$ & The distance of the pressure sensor node \\
$C_{S}$ & Cost to build undersea nodes \\
$N_{h}$ & Total number of undersea nodes, which also represent \\
$d^{i}$ & the total number of acoustic links \\
$\Theta_{T}$ & The length of the i-th acoustic link \\
$\Theta_{R}$ & Expected time constraint \\
& Link reliability constraint
\end{tabular}

\section{B. The Timeliness Constraint}

The timeliness constraint function, $T_{W a r n}()$, can be expressed as follows:

$$
T_{W a r n}(\Delta, \boldsymbol{d})=\tau_{t s u}(\Delta)-\tau_{n e t}(\Delta, \boldsymbol{d}) \geq \Theta_{T}
$$

where $\tau_{t s u}()$ is the tsunami travel time over $\Delta$ and $\tau_{n e t}()$ is the data delivery time from the pressure sensor to the control 
station ashore. Since fiber optic link is reliable and fast, the acoustic links bear the highest portion of the data delivery time.

Eq. (6) imposes a minimum distance of the pressure sensor node for a given $\Theta_{T}$. This minimum distance, denoted as $\Delta_{\min }$, can be derived if the data delivery time of the proposed infrastructure is made practically negligible. This would be possible if all-fiber deployment is chosen to cover $\Delta_{\min }$. This solution is considered as the most expensive solution. If either the epicenter or the pressure sensor distance is less than $\Delta_{\text {min }}$, then Eq 1 will never be satisfied. In our heuristic approach algorithm, $\Delta_{\min }$ holds important role for initial feasible solution.

1) The Data Delivery Time : This quantity is dictated by transmission delay, propagation delay, processing delay and retransmissions due to packet error. A less reliable link requires more time to complete a successful data delivery. Hence, we need to take the link reliability constraint function into account. Since the reliability constraint function implies a probabilistic type of quantity, the data delivery time, $\tau_{\text {net }}()$, is calculated as an average.

If $\mathcal{T}^{F}()$ represents the average of data delivery time in the fiber optic link and $\mathcal{T}^{S}()$ represent the average of data delivery time in the acoustic links, then the total data delivery time $\tau_{\text {net }}()$ is expressed as follow:

$$
\tau_{\text {net }}(\Delta, \boldsymbol{d})=\mathcal{T}^{F}(\Delta, \boldsymbol{d})+\mathcal{T}^{S}(\Delta, \boldsymbol{d})
$$

2) Tsunami Travel Time: To obtain tsunami travel time over $\Delta$, tsunami propagation speed needs to be determined. However, tsunami propagation speed is dictated by the sea depth. Given a sea depth of $h$, the tsunami speed, $v_{t s u}$, can be derived from $v_{t s u}=\sqrt{g \cdot h}$, where $g$ is the gravitational acceleration. To approximate tsunami travel time by taking the variation of sea depth into account, the pressure sensor distance $\Delta$ is divided into $K$ small intervals. The tsunami propagation speed in each interval is assumed to remain constant. Eq. (8) expresses the overall tsunami travel time as the sum of the travel time over $K$ intervals, where $x^{j}$ and $v_{t s u}^{j}$ denote the length and the tsunami propagation speed at $\mathrm{j}$-th interval. Indeed, larger $K$ results in more accurate result.

$$
\tau_{t s u}=\sum_{j=1}^{K} \frac{x^{j}}{v_{t s u}^{j}}
$$

\section{The Reliability Constraint}

The reliability constraint measures the probability of a successful data transmission. Given the data length of $m$, the probability of error-free received data at receiving node, $R()$, can be expressed as follows:

$$
R(\Delta, \boldsymbol{d})=\left(1-B E R_{r}(\Delta, \boldsymbol{d})\right)^{m}
$$

where $B E R_{r}$ is the probability of bit error. Since the fiber optic link offers very high reliability, the discussion focuses on the acoustic link reliability constraints expressed in Eq. (2). To obtain the bit error probability over an acoustic link $i$, a probability distribution function $\mathcal{F}(p)$ is specified. This probability distribution function represents the bit error characteristic in undersea environment. The probability function $\mathcal{F}(p)$ also depends on the modulation scheme. In section V, we will specify $\mathcal{F}(p)$ in more detail.

To apply this function, we use $p=\frac{E_{b}^{i}}{N_{0}^{i}}$ for the parameter. The $E_{b}^{i}$ and $N_{0}^{i}$ denote the energy per bit and equivalent white noise on acoustic link $i$. The following equation:

$$
E_{b}^{i}=\frac{1}{r_{s}} \cdot P_{t}^{i} \cdot A^{i}(\Delta, \boldsymbol{d}, f)
$$

provides the formulation to obtain the energy per bit on link $i$, where $P_{t}^{i}, A^{i}(), f$ and $r_{s}$ represent the node's transmission power, the acoustic channel loss, the acoustic wave frequency and the sensor transmission rate at link $i$, respectively.

To approximate the equivalent white noise energy, $N_{0}^{i}$, Eq. (11) is used. The equation divides the total noise power in the receiving node, $W(f, B)$, with the receiver's bandwidth $B$.

$$
N_{0}^{i}=\frac{1}{B} \cdot W(f, B)
$$

1) Acoustic Channel Loss: Acoustic channel loss is calculated as the total transmission loss inflicted by two main factors, namely spreading and absorption loss. A spreading loss is a signal attenuation as the acoustic signal travels further from the source, whereas the absorption loss is caused by the acoustic energy absorption as it traverses within sea water [10]. The acoustic energy absorption loss factor, denoted as $a(f)$, is frequency dependent. This paper incorporates this absorption loss into Bellhop model in order to increase the accuracy of the channel loss computation.

To quantify the absorption loss, $a(f)$, an empirical model, called as Thorp [11], is expressed as follows (in $\mathrm{dB}$ ) :

$$
a(f)=\frac{0.11 f^{2}}{1+f^{2}}+\frac{44 f^{2}}{4100+f^{2}}+2.75 \cdot 10^{-4} f^{2}+0.003
$$

where $f$ is in KHz. The equation above is still sufficiently valid for frequency above few hundreds of $\mathrm{Hz}$.

2) Sea Background Noise: Sea background noise, sometime is referred to as sea ambient noise, is inherent acoustic fields that may interfere the acoustic channel. Their characteristics and intensities are location-specific. Based on their sources, the ambient noise main contributors can be grouped as follows: water motion, including also the effects of surf, rain, hail and tides, man-made sources, including ship activity and marine life [8]. Each contributor causes a frequency-dependent impact to the acoustic channel. The ambient noise also increases in shallower depth.

A number of ambient noise studies and measurements have been made since 1945 in deep-water and open ocean areas [8]. The ambient noise spectra then are summarized and presented in a group of curves [8], [12] as shown in figure 2. Fig. 2 is also known as Wenz curve.

To obtain the total noise power $W(f, B)$ at the receiver with the receiving bandwidth of $B \mathrm{~Hz}$, the following equation,

$$
W(f, B)=\int_{B} w(f) d f
$$




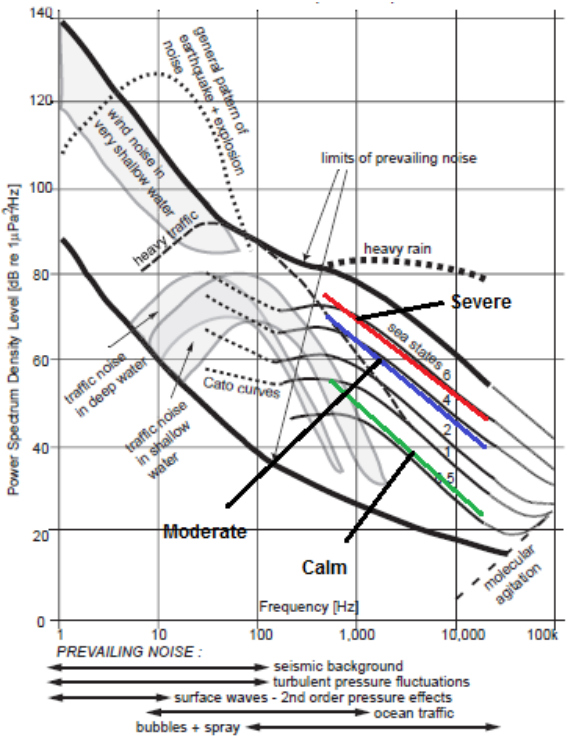

Fig. 2: Wenz curve, based on the work by [8]. This figure is taken from [12]

can be used. The term $w(f)$ represents the sea ambient noise spectrum function.

3) Bellhop Propagation Model: Underwater acoustic wave is a propagating mechanical wave that is created by the alternating compression and dilations of the medium [12]. The compression and the dilations result from the dynamic changes of medium pressure. Bellhop propagation model is a finite element approach which is based on ray tracing model. Although ray tracing model has coarser accuracy than other models such as Normal Mode or Parabolic Equation, it tends to improve in high frequency, especially above $1 \mathrm{KHz}$ [7].

Ray tracing model uses Helmholtz equation as the basis for derivation. If $\boldsymbol{x}=(x, y, z)$ denotes a point position expressed in Cartesian coordinate, then Helmholtz equation can be written as follow:

$$
\nabla^{2} p+\frac{\omega^{2}}{c^{2}(\boldsymbol{x})} p=-\delta\left(\boldsymbol{x}-\boldsymbol{x}_{0}\right)
$$

In underwater context, the term $p$ represents a function that quantifies the dynamic of medium pressure. The term $c(\boldsymbol{x})$ is the sound speed and $\omega$ is the angular frequency of the source located at $\boldsymbol{x}_{0}$. The solution of this equation results in two equations, called as eikonal equation and transport equations. Eikonal equation is used to compute the travel time along the ray, while transport equation is used to compute transmission loss.

Bellhop model describes the acoustic wave as a bundle of acoustic rays emitting from a source within a range of departure angles. Each acoustic ray will be computed independently. On their way to the receiver, acoustic rays may be reflected by either bottom, surface, or both. They may suffer from losses when being reflected by these boundaries. In addition to the reflection, the acoustic rays may also be refracted due to different sound speed across sea water depth. Under these circumstances, each ray's path is no longer in a straight line of trajectory and may differ one another.

Since each acoustic ray imposes a distinct path, they may result in different intensity and arrival time to each other at the receiving node. Therefore, transmission loss at the receiving node is calculated as the cumulative resultant of ray's intensity. A Bellhop program is used [13] to compute propagation loss and acoustic travel time between two nodes separated by the distance $d$. To run the program, undersea sound speed and sea floor profiles need to be supplied.

4) Undersea Sound Propagation Speed: Undersea sound speed depends on temperature, water salinity and the water pressure. Some empirical models explained in [14] express the underwater sound speed as the function of these three variables. One of those is formulated by Mackenzie as follow :

$$
\begin{aligned}
v_{a c}= & 1448.96+4.591 T-(5.304 e-2) T^{2}+(2.374 e-4) T^{3} \\
& +1.340(S-35)+(1.630 e-2) h+(1.675 e-7) h^{2} \\
& -(1.025 e-2) T(S-35)-(7.139 e-13) T h^{3}
\end{aligned}
$$

where $T$ is the sea water temperature at the depth $h$ and the salinity $S$. The temperature is measured in Celsius, the depth in meter, and the salinity in ppt (parts per thousand).

\section{The Heuristic ApProACH FOR Optimal SOlUtion}

Because the number of undersea sensors, $N_{h}$, is unknown a priori, we propose a heuristic approach. The heuristic starts with an initial feasible solution. We consider an all fiber optic of maximum length $\Delta_{\min }$ as the initial feasible solution of the optimization framework. Note, however, that such a solution is also the most expensive. Subsequently, the use of additional sensors, which results in the reduction of optic cable length, is explored to determine if the overall cost can be reduced. More specifically, the heuristic reduces the length of fiber optic by a certain amount and augments the infrastructure with acoustic sensors. The length of the removed fiber depends on the constraint imposed on the distance between the new acoustic sensor and the optic gateway. The timing constraint is also checked upon the removal of the fiber optic. This process continuous until the heuristic converges to a least-cost, constraint satisfying topology.

The approach has two ways of adding new undersea sensors, namely inbound and outbound. An inbound procedure can be seen as a direct substitution for the reduced fiber optic. The term "inbound" is given because the replacement progresses toward the shore. A maximization problem is solved to find the optimal inbound acoustic link length, denoted as $d_{i b}$, subject to the reliability and the timeliness constraints, $\Theta_{R}$ and $\Theta_{T}$, respectively. This process continuous until one of the the constraint is violated.

Note that repeatedly substituting the fiber optic with an inbound link may not always satisfy the timeliness constraint. To overcome the constraint violation, two techniques are used. The heuristic exploits these two techniques and select the better one. The first technique, which uses a binary search method, seeks to determine the optimal fiber optic extension within 


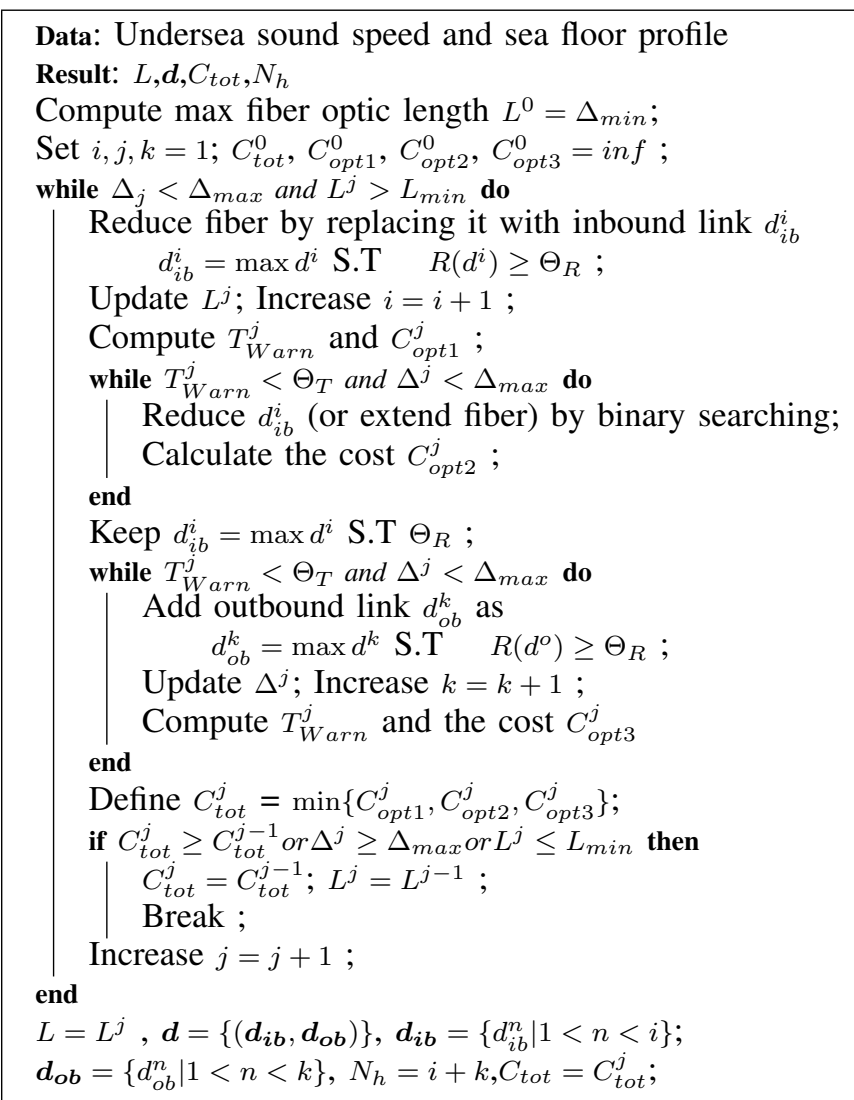

Fig. 3: Heuristic Approach

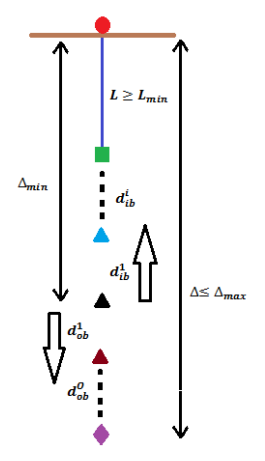

Fig. 4: Illustration of our heuristic approach

the latest added inbound link. The second technique adds one or more "outbound" acoustic links toward the tsunami origin. A maximization problem is then solved to find the optimal outbound acoustic link length, $d_{o b}$, subject to the reliability and the timeliness constraint.

The addition of an inbound link and possibly outbound links counts for one cycle. The cycle will be repeated if the implementation cost computed from the previous cycle

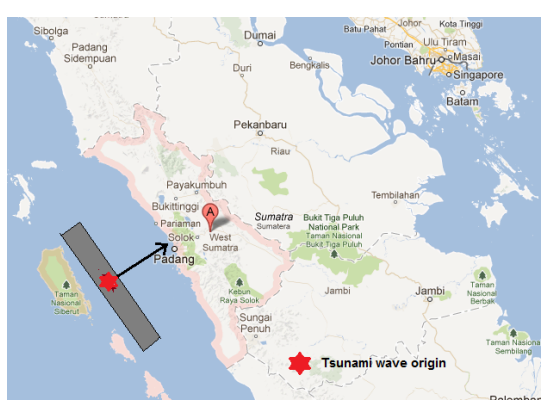

Fig. 5: Location of Padang, West Sumatra, Indonesia

TABLE II: Constants and Design Parameters

\begin{tabular}{l|l|l} 
Parameter & Value & Remark \\
\hline Tx Power Node & 40 Watt & \\
Modulation & Binary Freq. Shift Keying & \\
Data Link protocol & Stop and Wait & \\
$L_{\min }$ & $10 \mathrm{Km}$ & \\
$\Delta_{\max }$ & $90 \mathrm{Km}$ & Calm, in dB re $\mu \mathrm{Pa}^{2} / \mathrm{Hz}$ \\
$w(f)(1)$ & $110-20 \log _{10} \frac{f}{10}$ & Moderate, in $\mathrm{dB} \mathrm{re} \mu \mathrm{Pa}^{2} / \mathrm{Hz}$ \\
$w(f)(2)$ & $125-20 \log _{10} \frac{f}{10}$ & Severe, in dB re $\mu \mathrm{Pa}^{2} / \mathrm{Hz}$ \\
$w(f)(3)$ & $130-20 \log _{10} \frac{f}{10}$ & Packet length \\
$m$ & $120 \mathrm{bits}$ & Fiber transmission rate \\
$r_{f}$ & $200 \mathrm{Mbps}$ & See section V-G2 \\
$\gamma$ & 0.2 & \\
$\Theta_{T}$ & $\{15,16, \ldots, 20\}$ & Some time is written in $\%$, \\
$\Theta_{R}$ & $\{0.75,0.775, \ldots, 0.99,0.995,0.999\}$ & i.e $0.75=75 \%$ \\
$r_{s}$ & $\{40,80, \ldots, 440,480,520\}$ & in bit per second (bps) \\
$f$ & $\{4,6,8, \ldots, 262830\}$ & in KHz
\end{tabular}

is more expensive than the subsequent one. Otherwise, the approach will stop and returns the final result. These cycles may either eliminate completely the fiber optic or reach an infinite detection distance, $\Delta$. Given the fact that $L_{\min }$ and $\Delta_{\max }$ exist, the heuristic will stop whenever one of these boundaries is reached. Figure 3 and 4 illustrate our heuristic approach.

\section{CASE Study}

The main objective of the case study to derive an "optimal" configuration of a hybrid infrastructure for Padang City, West Sumatra, Indonesia, based on the developed framework. The framework will be applied under different sea environmental factors and design parameters. The region under consideration is shown in Fig. 5. The gray box in the figure represents the area of possible epicenters or tsunami origins. The underwater nodes and fiber optic would be deployed along the line pointing to the coast. The sea floor and the underwater sound speed profile data collected by [15] are used with permission. Different environment factors that impact background noise are also considered.

\section{A. Constants and Design Parameters}

Table II summarizes the constants and design parameters used in the case study. Fiber optic transmission rate is maintained to a fixed rate. We set a departure angle of the acoustic wave to 30 degrees and represent it in 120 acoustic rays. Three 
levels of ambient noise spectrum are assumed, namely calm, moderate, and severe. These assumptions reflect the windgenerated noise and associate them to the sea states scale as depicted in Wenz curve [12]. A "calm" assumption is associated to the sea state of 0.5. In this state, the noise is created by a calm sea with waves reaching up to 0.1 meter in average. A "moderate" level is referred to as the noise induced by the sea with sea state of 4 . At this level, the waves may reach a height of 3 meter in average. The highest noise level is attained when the sea is in "severe" state, which is caused by the wind that brings the sea waves up to 7 meters height. This state represents a sea scale of 6 in Wenz curve. In our case study, the underwater sensor transmits pressure data with some attributes, including the date, time, and battery status. We consider a 120 bit frame length to be sufficient to accommodate these data, including the frame overhead. We also assume that no forward error correction scheme is applied.

To deal with different ambient noise levels, this paper defines three design parameters, namely the link reliability constraint, acoustic carrier frequency and underwater transmission rate. This paper derives the optimal configuration and the implementation cost with these design parameters varied and analyze the results under different ambient noise conditions.

\section{B. Cost Function Assumption}

To limit the scope of analysis, the case study only focuses on linear cost function. Our discussion associates the variable $Q$ to two independent quantities. They mainly represent the physical attributes with respect to the infrastructure's component. More specifically, the fiber optic implementation cost is governed by the length of fiber optic $L$ and the fiber optic transmission rate $r_{f}$, whereas the underwater sensor network cost is dictated by the number of nodes $N_{h}$ and the node's transmission rate $r_{s}$. The following two equations express the cost function for the fiber optic and the undersea sensor network, respectively.

$$
\begin{gathered}
C_{F}=\phi_{f} \cdot r_{f} \cdot L \\
C_{S}=\phi_{s} \cdot r_{s} \cdot N_{h}
\end{gathered}
$$

To carry the cost analysis, the unit cost of sensor $\phi_{s}$ is normalized to the fiber optic unit cost $\phi_{f}$ such that $\phi_{s}=\gamma \phi_{f}$. The total cost, $C_{t o t}$, is expressed as the ratio of the cost of the most expensive solution. Without loss of generality, we assume that the initial deployment cost of the fiber optic and undersea sensors are the same.

\section{Formulation for Data Delivery Time}

Both the fiber optic and multi-hop acoustic links use automatic repeat request (ARQ) as the retransmission scheme. A stop and wait ARQ will be implemented in a per-hop basis. Consequently, the round trip transmission time of a packet and its associated acknowledgment can be expressed as follows:

$$
t_{r t}(d)=t_{m}+2 t_{p}(d)+t_{a}
$$

The parameter $t_{m}$ represents the data transmission time, $t_{p}(d)$ the acoustic propagation time traversing through a link of length $d$ and $t_{a}$ the acknowledgment packet transmission time. Given the reliability function, $R(\Delta, \boldsymbol{d})$, the average delay

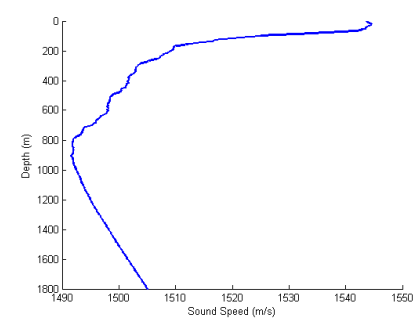

Fig. 6: Courtesy of BPPT [15]: Underwater Sound Speed around of our case study area

to transmit a packet and receive its acknowledgment can be expressed as follows:

$$
\mathcal{T}=\frac{1}{R(\Delta, \boldsymbol{d})} \cdot t_{r t}(d)
$$

Eq. (16) is applicable for both fiber optic and acoustic links. Based on Eq. (7), we can deduce a formulation for total data delivery time, $\tau_{\text {net }}()$, as follows :

$$
\tau_{n e t}(\Delta, \boldsymbol{d})=\frac{t_{t t}^{F}(L)}{R^{F}(\Delta, \boldsymbol{d})}+\sum_{i=1}^{N_{h}} \frac{t_{r t}^{S}\left(d^{i}\right)}{R^{i}(\Delta, \boldsymbol{d})}
$$

In the above equation, $t_{r t}^{F}$ denotes the round trip time transmission in the fiber optic link and $t_{r t}^{S}\left(d^{i}\right)$ is the round trip time transmission in acoustic link $i$. In the proposed heuristic, Eq. (17) is invoked each time the timeliness constraint is verified with a new value of $N_{h}$ being assigned for each invocation. In this paper, the fiber optic reliability is considered hight, i.e. $R^{F} \approx 1$. Thus, the data delivery time in fiber optic link, $\mathcal{T}^{F}$ is approximately equal to $t_{r t}^{F}$.

\section{Assumption on Bit Error Probability Distribution Function}

The case study uses binary frequency shift keying (BFSK) for the modulation scheme. To calculate bit error probability, this paper adopts the probability distribution function formulated in [16]. Let $\frac{E_{b}^{i}}{N_{0}^{i}}$ be the ratio of bit energy to equivalent white noise at link $i$. The bit error probability at link $i$ can be expressed as follows:

$$
B E R_{r}^{i}(\Delta, \boldsymbol{d})=\frac{v}{\Gamma(v)} \int_{0}^{\infty} u^{v-1}\left(2 v+u \frac{E_{b}^{i}}{N_{0}^{i}}\right) d u
$$

In the above equation, $\Gamma()$ represents the Gamma function, with $u=y^{2}$, where $y$ is the real part of complex value of signal amplitude random variable.

\section{E. Underwater Sound Speed and Sea Floor profile}

Figure 6 shows the characteristic of the underwater sound speed associated with the area under study. The figure shows that the speed of the acoustic wave drops rapidly in shallow waters of around 100 to 200 meters. The decrease in speed in deeper waters continue but at lower rate and re-bounce at a depth of about 800 meters. This profile is obtained by applying the measurement data collected by [15] into Eq. (14). Fig. 7 depicts the sea bottom profile along the line in Fig. 5. 


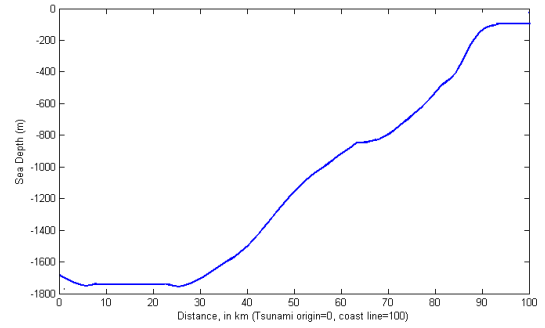

Fig. 7: Sea depth profile at area of interest

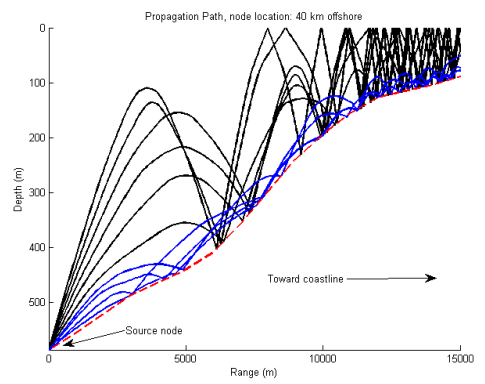

Fig. 8: Propagation path, generated using the Bellhop model

\section{F. Acoustic Propagation Path and Transmission Loss}

The varying underwater sound speed, combined with the sea floor profile, determines the propagation path of the acoustic wave. Fig. 8 shows the acoustic wave propagation generated using the Bellhop model, represented in 10 rays in total with departure angle of 10 degrees. The acoustic wave is emitted from a $40 \mathrm{~km}$ offshore-source with a frequency of $4 \mathrm{KHz}$. Due to the variation of undersea sound speed, the acoustic rays follow the paths depicted in Fig. 8. Fig. 9 illustrates the channel loss characteristics when two different acoustic carriers, with frequencies, $4 \mathrm{KHz}$ and $18 \mathrm{KHz}$, are emitted from two different locations. It can be observed that even when the acoustic waves are transmitted from the same distance, the acoustic waves with different frequencies and locations may produce different channel loss characteristics.

\section{G. Results}

1) Minimum Sensor Distance $\Delta_{m i n}$ : Given a set of values of the warning time, $\Theta_{T}$, listed in table II, the corresponding set of $\Delta_{\text {min }}$ is determined based on the sea floor profile data, using the equations described in section III-B1 and III-B2. These values represent the initial solutions of the proposed heuristic and are listed in table III.

\section{TABLE III: List of $\Delta_{\min }$}

\begin{tabular}{c|c|c|c|c|c|c}
$\begin{array}{c}\Theta_{T} \\
\text { (minutes) }\end{array}$ & 15 & 16 & 17 & 18 & 19 & 20 \\
\hline$\Delta_{\min }(\mathrm{Km})$ & 23.6 & 25.2 & 27.1 & 29.2 & 31.9 & 35.5
\end{tabular}

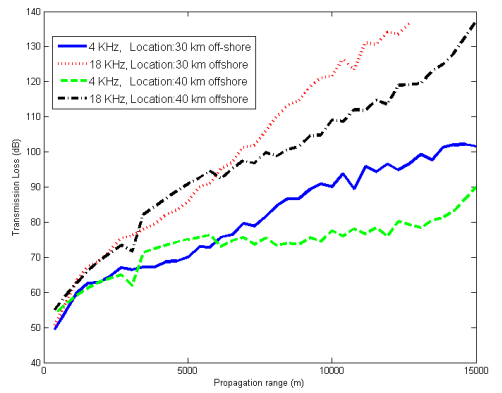

Fig. 9: Channel loss characteristics
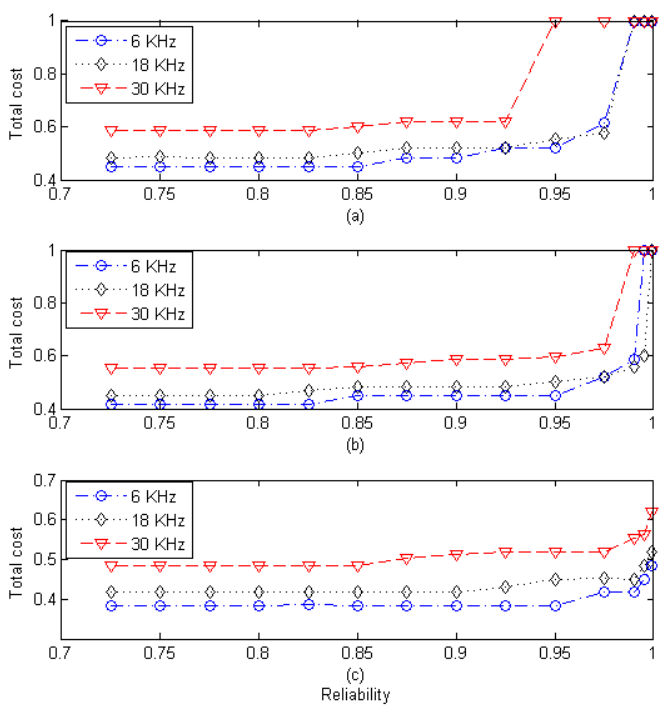

Fig. 10: Total cost for various reliability for 3 different frequencies with $\Theta_{T}=20$ minutes

2) The Results under Different Reliability Constraints: The focus is on investigating how the cost optimality can be achieved for different reliability constraints, frequencies and environmental factors. Three different acoustic wave frequencies, 6, 18 and $30 \mathrm{KHz}$ are selected to represent the low, middle and high frequency regions, respectively. In this case, we set $\Theta_{T}$ equals to 20 minutes and maintain the sensor's transmission rate at 120 bps. The remaining constants and parameters used in this experiment are listed in table II. The results are depicted by Fig. 10. The three sub figures, Fig 9(a), 9(b) and 9(c) show the results for severe, moderate and calm environments, respectively.

The results show that the optimal cost of the infrastructure does not increase significantly when the reliability constraint does not exceed 0.925. Consequently, if the desired reliability does not exceed 0.925 , a low-cost hybrid infrastructure using a low region of frequencies, is feasible. Achieving higher reliability, however, gives rise to trade-offs between the frequency used and the noise environment.

For example, for a reliability requirement of 0.99 , although 


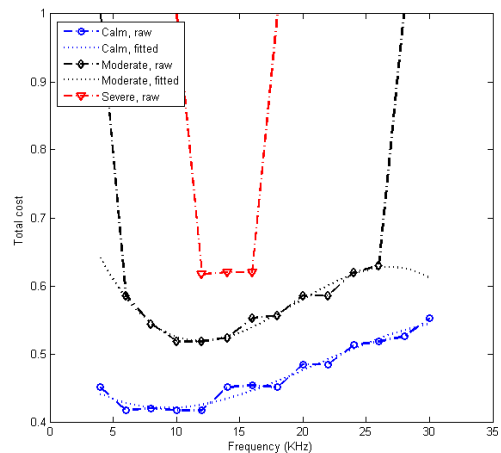

Fig. 11: Total cost for various frequency with $\Theta_{R}=0.99$

$6 \mathrm{KHz}$ frequency results in low-cost infrastructure in a calm environment, using a frequency of $18 \mathrm{KHz}$ achieves minimum cost, $C_{t o t}$, of approximately $2.93 \%$ less than the cost of an infrastructure operating with $6 \mathrm{KHz}$ in moderate environment. The difference becomes more notable for higher reliability requirements in severe environment. Rather than using undersea sensors operating with a frequency of $6 \mathrm{KHz}$, the optimization framework identifies the $18-\mathrm{KHz}$-based infrastructure a more cost-efficient infrastructure to achieve. The findings provide the basis to determine an optimal carrier frequency for different ambient noise levels. These findings will be discussed in detail in the following section.

3) The Results under Different Carrying Frequencies $f$ : Based on the findings observed in the previous section, a case analysis under different acoustic carrier frequencies and environment factors is the subject of this section. To this end, a reliability constraint of 0.99 is selected while retaining the timeliness constraint at 20 minutes. Fig. 11 shows the results of this study.

The result confirms the findings in section V-G2. Each ambient noise level imposes an optimal frequency range to use in order to achieve the minimum cost. In a calm environment, the optimal cost is achieved for low-range frequencies, namely 6 to $12 \mathrm{KHz}$. The frequency range shifts to a higher region as the environment becomes noisier.

In moderate environments, the optimal infrastructure cost is achieved for a frequency range of 10 to $16 \mathrm{KHz}$, whereas in severe environments, the frequencies to achieve optimal cost range from 12 to $18 \mathrm{KHz}$. Based on these results, an optimalcost infrastructure to meet the reliability and timeliness constraints in the three different environments can be achieved using a carrier frequency of $12 \mathrm{KHz}$. For our case study, the minimum cost infrastructure requires 4,7 and 9 nodes to be deployed for calm, moderate and severe environments, respectively. Table IV lists, for each environment, the distance, $d^{i}$, betweeen two consecutive undersea sensors and the length of the optic fiber associated with the configuration.

4) The Results under Different Sensor Tx Rates $r_{s}$ : This section focuses on investigating the impact to the results due to different undersea node's transmission rates. A frequency of $12 \mathrm{KHz}, 20$ minutes for the time constraint and 0.99 for the

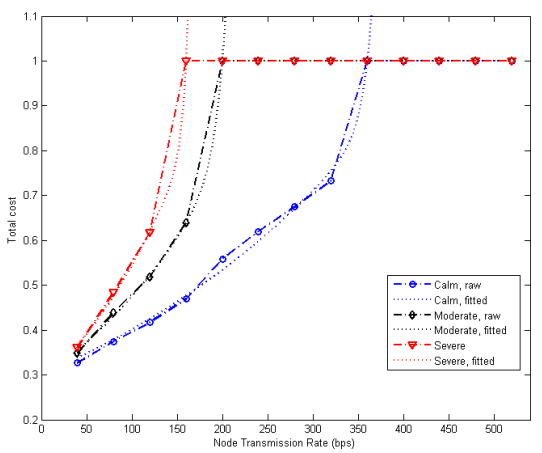

Fig. 12: Total cost for various node transmission rate under 3 different environments, with $\Theta_{R}=0.99$

TABLE IV: Optimal configuration at frequency of $12 \mathrm{KHz}$

\begin{tabular}{l|c|l|l|l|l|l|l|l|l|l}
\multirow{2}{*}{ Noise } & \multicolumn{8}{c|}{ Acoustic Link Length $(\mathbf{K m})$} & \multicolumn{1}{l}{$L$} \\
\cline { 2 - 9 } & $d^{1}$ & $d^{2}$ & $d^{3}$ & $d^{4}$ & $d^{5}$ & $d^{6}$ & $d^{7}$ & $d^{8}$ & $d^{9}$ & $(\mathbf{K m})$ \\
\hline Calm & 8.23 & 8.60 & 8.85 & 8.04 & - & - & - & - & - & \\
Moderate & 3.08 & 3.85 & 4.19 & 4.10 & 5.18 & 4.68 & 3.49 & - & - & 10 \\
Severe & 1.54 & 2.50 & 2.57 & 3.02 & 3.09 & 3.36 & 3.35 & 3.13 & 3.38 & 11.1
\end{tabular}

TABLE V: Optimal configuration with node's rate of $40 \mathrm{bps}$ and $160 \mathrm{bps}$

\begin{tabular}{c|c|c|c|c|c|c|}
\multirow{2}{*}{$\begin{array}{c}\text { Node's Rate } \\
\text { (bps) }\end{array}$} & \multicolumn{3}{|c|}{$\begin{array}{c}\text { Number of Sensor } \\
N_{h} \text { (unit) }\end{array}$} & \multicolumn{3}{c|}{ Fiber Optic Length } \\
\cline { 2 - 7 } & Calm & Moderate & Severe & Calm & Moderate & Severe \\
\hline 40 & 4 & 6 & 7 & 10 & 10 & 10 \\
160 & 4 & 7 & 0 & 10.27 & 11.51 & 35.5
\end{tabular}

reliability constraint are used. The result is show in Fig. 12.

A higher transmission rate requires a shorter acoustic link length to achieve the expected reliability. The cost increases exponentially and becomes more significant in severe noise environments. Table $\mathrm{V}$ shows the optimal results for the transmission rates of 40 and 160 bps. A 40 bps transmission rate doesn't incur significant increase in total cost when the environment becomes noisier. The cost increase is contributed by the number of undersea sensors. A more significant cost increase is observed for higher transmission rates. As the noise becomes worse, the cost increase is contributed by both the need of extra underwater sensors and the extension of the fiber optic cable. These results suggest that lower transmission rates is less sensitive to the impact of different ambient noises. However, selecting a very low transmission rate may violate the application data delivery time constraint, especially when larger amount of data are to be sent.

\section{CONCLUSION AND Future WORK}

The impact of tsunami on humans and the environment can be disastrous, causing severe damage to the infrastructure and great loss of lives, particularly in countries neighboring 
the Indian Ocean. Lessons learned from previous tsunamis reveal that a reliable and timely warning system increases the community resilience to tsunami disaster. To this end, we develop an optimization framework, which is used to derive a feasible and cost-effective infrastructure for NFT detection and warning. A case study is used to demonstrate the proposed approach and derive a feasible infrastructure for a region, which is prone to NFT, namely Padang City, West Sumatra, Indonesia. The results show that the proposed approach can lead to the derivation of an infrastructure that can guarantee 20 minute warning time and $99 \%$ data communication reliability.

The proposed framework can be used to provide insights and guidance related to the development and deployment of undersea tsunami detection and warning systems. As future work, the model can further be enhanced by incorporating multi-path loss models for a more realistic communication model and the development of energy management strategies to extend the lifetime of the undersea sensor subnetwork.

\section{ACKNOWLEDGMENT}

This material is based in part upon work supported by the National Science Foundation under Grants Number OCE 1331463: Hazards SEES Type 2: From Sensors to Tweeters: A Sustainable Socio-technical Approach for Detecting, Mitigating, and Building Resilience to Hazards. Any opinions, findings, and conclusions or recommendations expressed in this material are those of the authors and do not necessarily reflect the views of the National Science Foundation

\section{REFERENCES}

[1] UNESCO-IOC, “Tsunami glossary, 2008," IOC Technical Series, Paris, France, vol. 85, 2008.

[2] E. Sasorova, M. Korovin, V. Morozov, and P. Savochkin, "On the Problem of Local Tsunamis and Possibilities of Their Warning," Oceanology 2008, Pleiades Publishing Inc, vol. 48, no. 8, pp. 634-645, 2008.

[3] "NDBC- deep-ocean assessment and reporting of tsunamis (DART) description," [online] http://www.ndbc.noaa.gov/dart/dart.shtml, visited on April 2014

[4] L. Comfort, T. Znati, M. Voortman, Xerandy, and L. Freitag, "Early detection of near-field tsunamis using underwater sensor networks." Science of Tsunami Hazards, vol. 31, no. 4, 2012.

[5] C. Teng, S. Cucullu, S. M. Arthur, C. Kohler, B. Burnett, and L. Bernard, "Buoy vandalism by noaa national data buoy center," NOAA National Data Buoy Center1 University of Southern Mississippi2 report, June 2010.

[6] G. Schmitz, W. Rutzen, and W. Jokat, "Cable-based geophysical measurement and monitoring systems, new possibilities for tsunami earlywarnings," in Underwater Technology and Workshop on Scientific Use of Submarine Cables and Related Technologies, 2007. Symposium on. IEEE, 2007, pp. 301-304.

[7] M. Porter and Y.-C. Liu, "Finite-element ray tracing," Theoretical and Computational Acoustics, World Scientific Publishing Co, vol. 2, 1994

[8] G. M. Wenz, "Acoustic ambient noise in the ocean: Spectra and sources," The Journal of the Acoustical Society of America, vol. 34, no. 12, pp. 1936-1956, 1962. [Online]. Available: http://scitation.aip.org/content/asa/journal/jasa/34/12/10.1121/1.1909155

[9] J. McCloskey, A. Antonioli, A. Piatanesi, K. Sieh, S. Steacy, S. S. Nalbant, M. Cocco, C. Giunchi, J. D. Huang, and P. Dunlop, "Near-field propagation of tsunamis from megathrust earthquakes," Geophysical Research Letters, vol. 34, no. 14, 2007. [Online]. Available: http://dx.doi.org/10.1029/2007GL030494
[10] F. B. Jensen, Computational Ocean Acoustics. Springer Science \& Business Media, 1994

[11] L. M. Brekhovskikh and I. Lysanov, Fundamentals of ocean acoustics.

[12] C. Erbe, Underwater Acoustics: Noise and the Effects on Marine Mammals, a Pocket Handbook, 3rd ed. JASCO Applied Sciences, 2011.

[13] M. Porter, "The bellhop manual and userguide: Preliminary draft." [Online]. Available: http://oalib.hlsresearch.com/Rays/HLS-2010-1.pdf

[14] P. C. Etter, Underwater Acoustics Modeling and Simulation, 4th ed. CRC Press, 2013.

[15] B. Balai Teknologi Survei Kelautan, "Baruna jaya bppt 2014." [Online]. Available: http://barunajaya.bppt.go.id/index.php/en.html

[16] W.-B. Yang and T. C. Yang, "M-ary frequency shift keying communications over an underwater acoustic channel: Performance comparison of data with models," The Journal of the Acoustical Society of America, vol. 120 , no. 5, pp. 2694-2701, 2006.

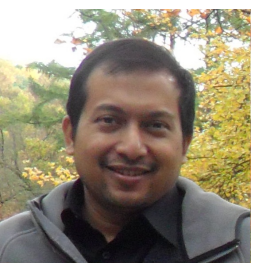

X. Xerandy received the Bachelor and Master degree from Bandung Institute of Technology, Indonesia, majoring in Electrical Engineering in 2006. He joined as the researcher in Agency of Technology Assessment and Application (BPPT), Indonesia since 2008. Currently, he is enrolled as a PhD student in School of Information Science, University Pittsburgh. His primary research is in telecommunication and networking. His current research project is to develop environment-aware undersea sensor network for tsunami disaster mitigation.

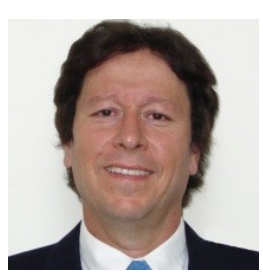

Taieb Znati is Professor in the Department of Computer Science, with a joint appointment in Computer Engineering at the School of Engineering. He served as the Director of the Computer and Network Systems Division at the National Science Foundation. $\mathrm{He}$ also served as a Senior Program Director for networking research at the National Science Foundation. In this capacity, $\mathrm{He}$ led the Information Technology Research Initiative, a cross-directorate research program. Dr. Znatis main research interests are in the design and analysis of evolvable, secure and resilient network architectures and protocols for wired and wireless communication networks. He is also interested in bio-inspired approaches to address complex computing and communications design issues that arise in large-scale heterogeneous wired and wireless networks. He is a recipient of several research grants from government agencies and from industry.

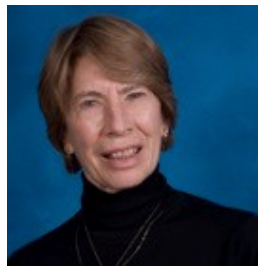

Louise K Comfort is Professor of Public and International Affairs and Director, Center for Disaste Management, University of Pittsburgh. She holds a B.A. in political science and philosophy, Macalester College, a M.A. in political science, University of California, Berkeley, and a Ph.D. in political science, Yale University. She is a Fellow, National Academy of Public Administration, and author or co-author of six books, including Designing Resilience: Preparing for Extreme Events, University of Pittsburgh Press, 2010 and Mega-Crises: Understanding the Prospects, Nature, Characteristics and the Effects of Cataclysmic Events, C. Thomas, 2012. Her primary research interests are in decision making under conditions of uncertainty and rapid change, and the uses of information technology to develop decision support systems for managers operating under urgent conditions. 